\title{
Prevalence and Factors Associated with Psychosocial Distress Among Adolescents in Beninese Schools in 2016
}

\author{
Agbohoui Houinato Olga ${ }^{1, *}$, Klikpo Tchédié Etdéchié Elvyre ${ }^{5}$, Hounkpatin Benjamin ${ }^{2}$, \\ Kpozehouen Alphonse ${ }^{3}$, Gbédji Kévin Yann ${ }^{1}$, Yoro Zohoun Inès ${ }^{1}$, Guédou Fernand ${ }^{1,4}$, \\ Houinato Dismand Stéphan ${ }^{1}$, Perrin René Xavier ${ }^{2}$
}

${ }^{1}$ Epidemiologic Laboratory for Chronic and Neurological Diseases (LEMACEN), Faculty of Health Sciences, University of Abomey Calavi, Cotonou, Benin

${ }^{2}$ Faculty of Health Sciences, University Hospital of Mother and Child Lagune (CHU MEL), University of Abomey Calavi, Cotonou, Benin

${ }^{3}$ Regional Institute of Public Health (IRSP), University of Abomey Calavi, Cotonou, Benin

${ }^{4}$ Health Center of Sexually Transmitted Infections (DIST), Health Center of Cotonou, Cotonou, Bénin

${ }^{5}$ Facullty of Health Sciences, National University Hospital Center of Psychiatrics of Cotonou, University of Abomey, Calavi, Cotonou, Benin

\section{Email address:}

oagbohoui@gmail.com (A. H. Olga),eklikpo@gmail.com (K. T. E. Elvyre), bhounkpatin@yahoo.fr(H. Benjamin), alphonse.kpozehouen@gmail.com (K.Alphonse),gbyann@gmail.com(G. K. Yann), yimeo2@yahoo.fr (Y.Z. Inès), guedaf@yahoo.fr(G. Fernand),dshouinato@gmail.com(H. D. Stéphan),perrinx@yahoo.fr(P. R. Xavier)

${ }^{*}$ Corresponding author

\section{To cite this article:}

Agbohoui Houinato Olga, Klikpo Tchédié Etdéchié Elvyre, Hounkpatin Benjamin, Kpozehouen Alphonse, Gbédji Kévin Yann, Yoro Zohoun Inès, Guédou Fernand, Houinato Dismand Stéphan, Perrin René Xavier. Prevalence and Factors Associated with Psychosocial Distress Among Adolescents in Beninese Schools in 2016. World Journal of Public Health. Vol. 4, No. 3, 2019, pp. 55-64. doi: 10.11648/j.wjph.20190403.12

Received: July 22, 2019; Accepted: August 29, 2019; Published: September 17, 2019

\begin{abstract}
Context: Adolescence is the period during which a person experiences significant physical, biological and emotional changes. The lack of attention to mental well- being of adolescents may have adverse consequences on their mental health. The objective of this study was to estimate the prevalence of psychological distress in schooled adolescents and the factors associated with it. Methods: This was a cross-sectional study conducted in 2016 and involving 3,841 adolescents of 10 to 19 years from 40 secondary schools in Benin. Participants were selected using a 2-level random sampling technique. Data were collected through a self-administered questionnaire and were analyzed using Epi Info and Stata softwares. Associated factors were investigated by a logistic regression. Results: Mean age was $16.54 \pm 0.04$ years and sex ratio was 1.16 . The prevalence of psychosocial distress was $10.8 \% \mathrm{CI}_{95 \%}$ : 9.44-12.15). Multivariate analysis showed a significant association between bullying, friendships, the class attended, the place of residence and the person with whom the adolescent lives. Teenagers who had been bullied were almost twice as likely to develop psychosocial distress. Having a close friend was a protective factor from psychosocial distress. Being in examination years $\left(9^{\text {th }}\right.$ grade or final year of high school $)$ increased by 4 times the risk of developing psychosocial distress. Conclusion: Psychosocial distress, previously mostly observed in developed countries, is now also reaching emerging countries. The implementation of urgent actions for the prevention of psychosocial distress is imposed on decision-makers.
\end{abstract}

Keywords: Prevalence, Psychosocial Distress, Adolescent, School Environment, Benin

\section{Introduction}

The stable economic development of a country depends on the level of education, the state of health and the optimal well-being of adolescents and young people.

Adolescence is the period during which a person undergoes physical, biological and emotional changes [1]. During this period, adolescents may suffer from various forms of problems, psychosocial dysfunctions, and conflicts 
that may affect their normal psychosocial development.

Half of the mental disorders registered begin before the age of 14 and $75 \%$ begin at age of $24[2,3]$. Studies in Canada and the United States have demonstrated that adolescent mental health is a public health issue $[4,5]$. In developing countries, such as Zambia, Nepal, or other South Asian countries, mental health problems are occurring more acutely than in developed countries [5].

The lack of attention towards adolescents' mental wellbeing, while in a key phase of socialization, may have a lifelong impact on mental health and reduce the capacity of societies' socio-economic productivity [3,6]. According to Bista \& al. the adequate psychosocial development of the adolescent results in good school performances, a good physical health and an adequate social, emotional and psychological health [1]. This ultimately helps to reduce the risks of psychosocial and behavioral issues, violence, criminality, teenage pregnancy, drugs use and alcohol abuse [7]. Detecting a psychological distress issue in the beginning of adolescence could be relevant to enhance the individual's quality of life. However, very few studies on the phenomenon were conducted in Benin; these studies only gave a description of the phenomenon and did not extend to the consideration of risks factors related to psychosocial distress in adolescents [8].

This study aimed to determine the prevalence and factors associated with the psychological distress of schooled adolescents in Benin in 2016. This should highlight hidden and neglected public health issues and help drawing the decision-makers attention on the necessity of setting up mental health programs for adolescents.

\section{Methodology}

\subsection{Description of the Survey and the Study Population}

We analyzed data from the national survey on the current status and factors associated with adolescent health in schools in Benin in 2016. This was a cross-sectional study targeting 10 to 19 -year-olds children of both sex and enrolled from first to seventh (final) grades of secondary school. The number of subjects required was calculated by the Schwartz formula and was 3,841 . The sampling was conducted with the technical support of CDC Atlanta, using a 2-degree random sampling technique with a probability proportional to the size of the schools and classes.

The first level consisted in selecting 40 out of 1494 public and private secondary schools, with a probability proportional to the size of the institutions. At the second level, within each selected institution, a census of all classes was conducted. The choice of classes to be investigated was randomly made from a list of random numbers, preestablished and made available by CDC Atlanta and WHO. This list of numbers varied from a school to another. The number of classes to be surveyed per institution was proportional to the total number of classes available in these selected institutions. Finally, all adolescents of eligible classes present during the survey were included in the study.

The data collection tool was an adapted self-administered WHO questionnaire that included demographic and foods behavior data.

A standardized questionnaire was used for data collection. Investigative administrators trained after the informed consent of adolescents or their parents guided them to complete the questionnaire.

\subsection{Variables}

\subsubsection{Dépendent Variable}

The dependent variable was the psychosocial distress defined using four mental health indicators that were loneliness, anxiety, sadness or worry and having seriously thought about a suicide plan. This definition was based on previous researches on a population of similar schooled adolescents $[9,10]$. An adolescent who had answered "Yes" to at least 2 mental health indicators was considered under psychosocial distress.

\subsubsection{Independent Variables}

The independent variables were: age; gender; area of residence; class attended; type of school (public or private); bullying; school fighting; consumption of alcohol; drug use; physical activity; relationship with parents; parental support and supervision and friendship.

\subsection{Data Analysis}

The data were entered with the Excel software and analyzed using the STATA 13.0 (Stata Corporation, College Station, Texas, United State) software. The qualitative variables were estimated in proportion with their 95\% confidence intervals $(95 \% \mathrm{CI})$ and the quantitative ones in means with \pm standard deviation (SD).

Associations between qualitative variables were studied using the Pearson Chi2 test. The strength of the associations between the dependent variable ("psychosocial distress ") and the independent variables was assessed by calculating Odds Ratios (OR) followed by their $95 \%$ CI.

Since psychosocial distress is not equally perceived by a male and a female adolescent, we first presented our findings for all the adolescents, then according to their gender.

A logistic regression was used to evaluate the influence of the explanatory variables on the psychosocial distress. All statistically significant variables at a p-value $<0.20$ in the univariate analysis were included in the multivariate models and a stepwise modeling procedure was adopted to select the variables that were used to build the final model. Only variables with a p-value of less than 0.05 have been retained in the final model. To take into account the effect of the sampling procedure used, we weighted all our analyses [11]. The adequacy of the model was verified using the Hosmer Lemshow test.

\subsection{Ethical Consideration}

The protocol was approved by the Institutional Research 
Ethics Committee of the Institute of Applied Biological Sciences (CER-ISBA). Legally major students (18 years or older) provided their informed consent while the minor ones (less than 18 years) provided their informed assent along with their parents informed consent. All data were anonymous.

\section{Results}

Our analyses included 3,968 school adolescents, which corresponds to a response rate of $78.0 \%$ of the sampled

\section{population.}

Table 1 and figure 1 shows the distribution of adolescents according to general characteristics. The average age of the 3,968 adolescents included in the study was $16.54 \pm 0.04$ years and the sex ratio male/female of 1.16 . They lived mainly in urban areas (60.50\%) and $68.90 \%$ were in public schools. More than one in two teens had lived with their parents in the last 30 days prior to the survey (Figure 1). The proportion of adolescents being bullied was $38.60 \%$ while that of those having at least one close friend was $89.30 \%$.

Table 1. Distribution of school adolescents by general characteristics in Benin in 2016.

\begin{tabular}{|c|c|c|}
\hline & Frequency & $\%$ (weighted) \\
\hline \multicolumn{3}{|l|}{ Age } \\
\hline$<15$ years & 1,333 & 13.28 \\
\hline$\geq 15$ years & 2,620 & 86.72 \\
\hline \multicolumn{3}{|l|}{ Gender } \\
\hline Male & 2,127 & 72.92 \\
\hline Female & 1,826 & 27.08 \\
\hline \multicolumn{3}{|l|}{ Being bullied } \\
\hline 0 day & 2,224 & 61.4 \\
\hline $1-30$ days & 1,705 & 38.55 \\
\hline \multicolumn{3}{|l|}{ Fight } \\
\hline 0 time & 2,651 & 76.11 \\
\hline 1 or more times & 1,310 & 23.89 \\
\hline \multicolumn{3}{|l|}{ Alcohol consumption } \\
\hline 0 day & 2,170 & 51.66 \\
\hline 1 or more days & 1,745 & 48.34 \\
\hline \multicolumn{3}{|l|}{ Tobacco consumption } \\
\hline Yes & 236 & 8.00 \\
\hline No & 3,728 & 92.00 \\
\hline \multicolumn{3}{|l|}{ Illicite Drug consumption } \\
\hline Yes & 142 & 4.70 \\
\hline No & 3,822 & 95.3 \\
\hline \multicolumn{3}{|c|}{ Regular Practice of Physical Activity } \\
\hline$<3$ days & 1,884 & 43.58 \\
\hline $3-7$ days & 2,071 & 56.42 \\
\hline \multicolumn{3}{|l|}{ Parental supervision } \\
\hline Never. rarely or sometimes & 2,382 & 67.59 \\
\hline Most of the time or always & 1,572 & 32.41 \\
\hline \multicolumn{3}{|l|}{ Parental support } \\
\hline Never rarely or sometimes & 2,447 & 67.53 \\
\hline Most of the time or always & 1,504 & 32.47 \\
\hline \multicolumn{3}{|c|}{ Relationship with parents/guardians } \\
\hline Yes & 2,375 & 64.21 \\
\hline No & 1,554 & 35.79 \\
\hline \multicolumn{3}{|l|}{ Friendship } \\
\hline 0 friend & 452 & 10.72 \\
\hline 1 or more & 3,499 & 89.28 \\
\hline \multicolumn{3}{|l|}{ Class attended } \\
\hline $1^{\mathrm{st}}$ & 653 & 0.34 \\
\hline $2^{\text {nd }}$ & 794 & 0.17 \\
\hline $3^{\text {rd }}$ & 915 & 30.06 \\
\hline $4^{\text {th }}$ & 657 & 30.72 \\
\hline $5^{\text {th }}$ & 408 & 12.44 \\
\hline $6^{\text {th }}$ & 174 & 11.66 \\
\hline $7^{\text {th }}$ & 367 & 14.62 \\
\hline \multicolumn{3}{|l|}{ Type of school } \\
\hline Public & 1,025 & 31.12 \\
\hline Private & 2,928 & 68.88 \\
\hline \multicolumn{3}{|l|}{ Area of residence } \\
\hline Rural & 918 & 395 \\
\hline Urban & 2,272 & 60.5 \\
\hline \multicolumn{3}{|l|}{ Department } \\
\hline Alibori & 434 & 17.78 \\
\hline Atacora & 305 & 8.87 \\
\hline
\end{tabular}




\begin{tabular}{lll}
\hline & Frequency & \% (weighted) \\
\hline Atlantique & 489 & 9.67 \\
Borgou & 549 & 14.77 \\
Collines & 119 & 4 \\
Couffo & 57 & 0.98 \\
Donga & 86 & 2.54 \\
Littoral & 330 & 7.64 \\
Mono & 305 & 7.06 \\
Oueme & 571 & 11.06 \\
Plateau & 122 & 3.21 \\
Zou & 551 & 12.42 \\
\hline
\end{tabular}

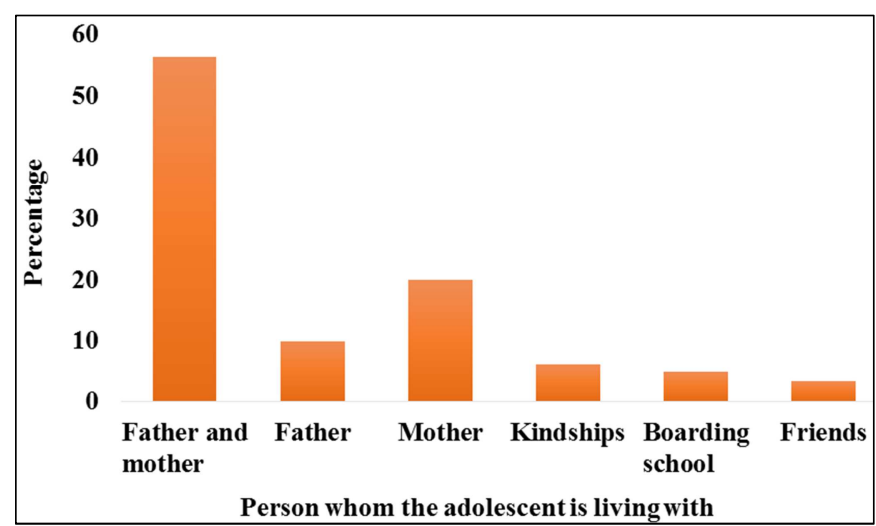

Figure 1. Distribution of school adolescents according to the person with whom they lived the last 30 days preceding the survey in Benin in 2016.

Table 2 Shows the characteristics of adolescents having suffered from psychosocial distress in schools.

Table 2. Characteristics of adolescents having suffered from psychosocial distress in school environment in Benin 2016.

\begin{tabular}{|c|c|c|c|}
\hline & Female adolescents & Male adolescents & Overall \\
\hline & \% (weighted) & \% (weighted) & $\%$ (weighted) \\
\hline \multicolumn{4}{|l|}{ Age } \\
\hline$<15$ years & 7.90 & 4.80 & 5.58 \\
\hline$\geq 15$ years & 11.72 & 11.5 & 11.55 \\
\hline \multicolumn{4}{|l|}{ Gender } \\
\hline Male & & 10.70 & 10.70 \\
\hline Female & 11.07 & & 11.07 \\
\hline \multicolumn{4}{|l|}{ Being bullied } \\
\hline 0 day & 7.46 & 9.52 & 8.99 \\
\hline $1-30$ days & 16.29 & 12.83 & 13.81 \\
\hline \multicolumn{4}{|l|}{ Fight } \\
\hline 0 time & 10.84 & 10.62 & 10.68 \\
\hline 1 or more times & 12.17 & 11.03 & 11.28 \\
\hline \multicolumn{4}{|l|}{ Alcohol consumption } \\
\hline 0 day & 9.54 & 10.18 & 9.99 \\
\hline 1 or more days & 12.76 & 11.08 & 11.50 \\
\hline \multicolumn{4}{|l|}{ Tobacco consumption } \\
\hline Yes No & 10.46 & 15.36 & 14.97 \\
\hline \multicolumn{4}{|l|}{ Drug consumption } \\
\hline Yes No & 11.08 & 10.19 & 10.45 \\
\hline \multicolumn{4}{|c|}{ Regular Practice of Physical Activity } \\
\hline$<3$ days & 4,90 & 12.13 & 11.58 \\
\hline 3-7 days & 11.15 & 10.63 & 10.77 \\
\hline \multicolumn{4}{|l|}{ Parental supervision } \\
\hline Never. rarely or sometimes & 11.98 & 11.60 & 11.72 \\
\hline Most of the time or always & 10.17 & 10.12 & 10.13 \\
\hline \multicolumn{4}{|l|}{ Parental support } \\
\hline Never rarely or sometimes & 10.60 & 10.55 & 10.56 \\
\hline Most of the time or always & 11.59 & 10.96 & 11.18 \\
\hline \multicolumn{4}{|c|}{ Relationship with parents/guardians } \\
\hline Yes & 11.64 & 11.15 & 11.28 \\
\hline No & 9.74 & 9.64 & 9.66 \\
\hline \multicolumn{4}{|l|}{ Friendship } \\
\hline 0 friend & 11.38 & 11.42 & 11.41 \\
\hline
\end{tabular}




\begin{tabular}{|c|c|c|c|}
\hline & Female adolescents & Male adolescents & Overall \\
\hline & $\%$ (weighted) & $\%$ (weighted) & $\%$ (weighted) \\
\hline 1 or more & 10.14 & 9.44 & 9.64 \\
\hline \multicolumn{4}{|l|}{ Class attended } \\
\hline $1^{\mathrm{st}}$ & 15.78 & 17.52 & 16.88 \\
\hline $2^{\text {nd }}$ & 10.28 & 10.07 & 10.12 \\
\hline \multicolumn{4}{|l|}{$3^{\text {rd }}$} \\
\hline $4^{\text {th }}$ & 2.84 & 1.17 & 1.75 \\
\hline $5^{\text {th }}$ & 6.01 & 4.84 & 5.39 \\
\hline $6^{\text {th }}$ & 8.68 & 8.04 & 8.22 \\
\hline $7^{\text {th }}$ & 15.99 & 11.19 & 12.57 \\
\hline Type of school & 7.65 & 12.17 & 11.07 \\
\hline Public & 6.68 & 8.01 & 7.68 \\
\hline Private & 11.62 & 15.79 & 14.83 \\
\hline \multicolumn{4}{|c|}{ Area of residence } \\
\hline Rural & 16.4 & 12.79 & 13.31 \\
\hline Urban & 9.09 & 9.39 & 9.29 \\
\hline \multicolumn{4}{|l|}{ Department } \\
\hline Alibori & 13.12 & 8.67 & 9.93 \\
\hline Atacora & 10.78 & 12.1 & 11.88 \\
\hline Atlantique & 8.51 & 6.25 & 7.12 \\
\hline Borgou & 11.39 & 9.86 & 10.32 \\
\hline Collines & 0.13 & 19.11 & 16.78 \\
\hline Couffo & $7.10^{-4}$ & 22.15 & 17.57 \\
\hline Donga & $9.10^{-4}$ & 11.9 & 11.15 \\
\hline Littoral & 10.68 & 8.24 & 8.99 \\
\hline Mono & 6.73 & 8.79 & 8.48 \\
\hline Oueme & 11.03 & 10.64 & 10.78 \\
\hline Plateau & 7.79 & 13.14 & 11.78 \\
\hline Zou & 18.33 & 15.30 & 16.12 \\
\hline
\end{tabular}

\subsection{Prevalence and Factors Associated with Psychosocial Distress}

The prevalence of psychosocial distress in adolescents was $10.8 \%\left(\mathrm{CI}_{95 \%}: 9.44-12.15\right)$ and Table 3 summarizes the factors associated with psychosocial distress prevalence in univariate and multivariate analysis.

\subsection{Univariate Analysis}

From the univariate analysis several factors were associated with psychosocial distress among adolescents. Adolescents aged 15 and over were more suffering from psychosocial distress $(11.6 \%)$ and were twice more likely to suffer from psychosocial distress than adolescents under 15 years of age $\left(\mathrm{OR}=2.08 \mathrm{CI}_{95 \%}\right.$ 1.32-3.29). Adolescents who reported having been bullied were almost two times more likely to suffer from psychosocial distress than those who did not $\left(\mathrm{OR}=1.62 \mathrm{CI}_{95 \%}\right.$ 1.22-2.15). Having at least one close friend was a protective factor for adolescents not to suffer from psychosocial distress $\left(\mathrm{OR}=0.55 \quad \mathrm{CI}_{95 \%} \quad 0.37-0.82\right)$. Adolescents attending examination classes such as ninth grade or final year of high school were more likely to be psychologically distressed $\left(\mathrm{OR}=8.05 \mathrm{CI}_{95 \%}\right.$ 3.73-17. 36; $\mathrm{OR}=9.74 \mathrm{CI}_{95 \%}$ 4.43-21.43). The urban environment was a protective factor from psychosocial distress $\left(\mathrm{OR}=0.66 \mathrm{CI}_{95 \%}\right.$ 0.48-0.91. As for the person with whom the adolescent lived most, adolescents living in boarding schools were two times more likely to develop psychosocial distress than those living with their parents $\left(\mathrm{OR}=2.34 \mathrm{CI}_{95 \%} 1.38-3.97\right)$.

Girls seemed to be more suffering from psychosocial distress than boys $(11.7 \%$ versus $10.7 \%)$, but that difference was not statistically significant $(p=0.78)$. However the gender has modified the association between several factors and psychological distress.

Thus, the association between some factors and psychological distress was stronger among boys than girls. This was the case of the age (15 and older versus younger than 15) with an $\left(\mathrm{OR}=2.57 \mathrm{CI}_{95 \%} 1.28-5.17\right)$ for boys versus $\left(\mathrm{OR}=1.54 \mathrm{CI}_{95 \%}\right.$ 0.89-2.67) for girls, the type of school (public or private) with $\left(\mathrm{OR}=1.58 \mathrm{CI}_{95 \%}\right.$ 1.02-2.46) for boys versus $\left(\mathrm{OR}=0.80 \mathrm{CI}_{95 \%} 0.54-1.19\right)$ for girls.

In the contrary, the association between having been bullied and psychological distress was more noticeable among girls $\left(\mathrm{OR}=2.41 \mathrm{CI}_{95 \%}\right.$ 1.62-3.58) than boys $\left(\mathrm{OR}=1.39 \mathrm{CI}_{95 \%} 0.96-\right.$ 2.01) as was that between attended class and psychological distress with $\left(\mathrm{OR}=10\right.$. 64; $\mathrm{CI}_{95 \%}$ 3.78-29.95) among boys versus $\left(\mathrm{OR}=15.83 \mathrm{CI}_{95 \%}\right.$ 1.30-15.51) among girls for being in $9^{\text {th }}$ grade and $\left(\mathrm{OR}=6.51 \mathrm{CI}_{95 \%} 2.05-20.66\right)$ versus $(\mathrm{OR}=4.50$ $\mathrm{CI}_{95 \%}$ 2.05-20.66) for being in the final year of high school.

Finally, for some factors the strength of the association was similar for both boys and girls. Examples included having at least one close friend, whose protective effect was equally observed among both girls and boys with $(\mathrm{OR}=0.61$ $\left.\mathrm{CI}_{95 \%} 0.37-0.99\right)$ and $\left(0.52 \mathrm{CI}_{95 \%} 0.30-0.90\right)$ respectively and living in urban environment whose protective effect was similar among both girls $\left(\mathrm{OR}=0,51 \quad \mathrm{CI}_{95 \%} 0.31-0.81\right)$ and boys $\left(\mathrm{OR}=0.70 \mathrm{CI}_{95 \%}\right.$ 0.47-1.05).

\subsection{Multivariate Analysis}

The multivariate analysis revealed a significant association 
between bullying $\left(\mathrm{aOR}=1.53 \quad \mathrm{CI}_{95 \%}\right.$ 1.08-2.17), having at least one close friend $\left(\mathrm{aOR}=0.58 \mathrm{CI}_{95 \%} 0.36-0.94\right)$, being in an examination class $\left(4^{\text {th }}\right.$ year of high school $)$ with $(\mathrm{aOR}=$ $\left.3.87 \mathrm{CI}_{95 \%} 1.11-13.55\right)$ or in the $7^{\text {th }}$ year with $(\mathrm{aOR}=4.71$ $\mathrm{CI}_{95 \%}$ 1.32-16.76), living in a boarding house (aOR $=2.34$ $\mathrm{CI}_{95 \%}$ 1.26-4.35) and psychosocial distress.

As in the univariate analysis, some of these associations were modified by the gender. The association between attended classes was greater among boys $\left(\mathrm{aOR}=6.63 \mathrm{CI}_{95 \%}\right.$ 1.14-38.57);

$\mathrm{aOR}=8.72 \mathrm{CI}_{95 \%}$ 1.52-50.08) than among girls $(\mathrm{aOR}=$
$1.95 \mathrm{CI}_{95 \%}$ 0.90-5.48; aOR $=1.73 \mathrm{CI}_{95 \%}$ 0.66-4.53). Likewise, the association between living in boarding house and psychological distress was stronger for boys $(\mathrm{aOR}=2.78$ $\mathrm{CI}_{95 \%}$ 1.40-5.54) than for girls $\left(\mathrm{aOR}=2.34 \mathrm{CI}_{95 \%}\right.$ 1.26-4.35).

In the contrary, the association between some factors and the psychological distress was stronger among girls than boys. The effect of bulling was more noticeable among girls $\left(\mathrm{aOR}=2.40 \mathrm{CI}_{95 \%}\right.$ 1.47-3.93). The fact to have at least one close friend was also more noticeable among girls $(\mathrm{aOR}=$ $\left.0.56 \mathrm{CI}_{95 \%} 0.31-0.99\right)$.

Table 3. Association between psychosocial distress and independent variables among school adolescents in Benin in 2016: univariate and multivariate analyses.

\begin{tabular}{|c|c|c|c|}
\hline & \multicolumn{3}{|l|}{ Univariate analysis } \\
\hline & Overall & female adolescents & Male adolescents \\
\hline Age & 0.001 & 0.11 & 0.007 \\
\hline$<15$ ans & 1 & 1 & 1 \\
\hline$\geq 15$ ans & $2.08(1.32-3.29)^{* *}$ & $1.54(0.89-2.67)$ & $2.57(1.28-5.17)^{*}$ \\
\hline Beingbullied & 0.0008 & $10^{-4}$ & 0.07 \\
\hline 0 day & 1 & 1 & 1 \\
\hline $1-30$ days & $1.62(1.22-2.15)^{* * *}$ & $2.41(1.62-3.58)^{* * *}$ & $1.39(0.96-2.01)$ \\
\hline Friendship & 0.003 & 0.04 & 0.02 \\
\hline 0 friend & 1 & 1 & 1 \\
\hline 1 or more & $0.55(0.37-0.82)^{* *}$ & $0.61(0.37-0.99)^{* *}$ & $0.52(0.30-0.90)^{*}$ \\
\hline Class attended & $10^{-4}$ & 0.001 & 0 \\
\hline $1^{\text {st }}$ & 1 & 1 & 1 \\
\hline $2^{\text {nd }}$ & $3.18(1.29-7.87)^{* *}$ & $2.18(0.56-8.51)$ & $4.29(1.26-14.58)^{* *}$ \\
\hline $3^{\text {rd }}$ & $5.02(2.31-10.89)^{* * *}$ & $3.25(1.01-10.40) * *$ & $7.38(2.61-20.83)^{* * *}$ \\
\hline $4^{\text {th }}$ & $8.05(3.73-17.36)^{* * *}$ & $6.51(2.05-20.66)^{* * *}$ & $10.64(3.78-29.95) * * *$ \\
\hline $5^{\text {th }}$ & $6.97(3.13-15.52)^{* * *}$ & $2.83(0.79-10.16)$ & $11.69(4.10-33.36)^{* * *}$ \\
\hline $6^{\text {th }}$ & $4.66(1.72-12.61)^{* *}$ & $2.45(0.63-9.49)$ & $7.34(2.03-26.61)^{* *}$ \\
\hline $7^{\text {th }}$ & $9.74(4.43-21.43)^{* * *}$ & $4.50(1.30-15.51)^{* *}$ & $15.83(5.61-44.68) * * *$ \\
\hline Type of school & 0.14 & 0.27 & 0.04 \\
\hline Public & 1 & 1 & 1 \\
\hline Private & $1.26(0.92-1.72)$ & $0.80(0.54-1.19)$ & $1.58(1.02-2.46)^{*}$ \\
\hline Area of residence & 0.01 & 0.004 & 0.08 \\
\hline Rural & 1 & 1 & 1 \\
\hline Urban & $0.66(0.48-0.91)^{* *}$ & $0.51(0.31-0.81)^{* *}$ & $0.70(0.47-1.05)$ \\
\hline Person with whom the teenager most lived & 0.04 & 0.73 & 0.04 \\
\hline Father and mother & 1 & 1 & 1 \\
\hline Father & $1.04(0.63-1.71)$ & $0.75(0.33-1.17)$ & $1.15(0.62-2.09)$ \\
\hline Mother & $1.06(0.74-1.53)$ & $0.80(0.49-1.29)$ & $1.21(0.73-1.95)$ \\
\hline kinships & $1.13(0.59-2.14)$ & $1.43(0.60-3.39)$ & $1.09(0.48-2.41)$ \\
\hline Boarding school & $2.34(1.38-3.97)^{* *}$ & $1.22(0.33-4.42)$ & $2.63(1.44-4.72)^{* * *}$ \\
\hline Friends & $0.62(0.25-1.53)$ & $0.61(0.18-2.05)$ & $0.61(0.18-2.06)$ \\
\hline Alcohol comsuption & 0.28 & 0.1 & 0.61 \\
\hline 0 day & 1 & 1 & 1 \\
\hline 1 or more days & $1.17(0.87-1.55)$ & $1.38(0.93-2.04)$ & $1.09(0.76-1.58)$ \\
\hline Tobacco comsuption & 0.12 & 0.91 & 0.10 \\
\hline No & 1 & 1 & 1 \\
\hline Yes & $1.50(0.89-2.55)$ & $0.93(0.27-3.20)$ & $1.59(0.90-2.81)$ \\
\hline Department & & $10^{-4}$ & \\
\hline Alibori & 1 & 1 & 1 \\
\hline Atacora & $1.22(0.70-2.12)$ & $0.80(0.30-2.09)$ & $1.45(0.73-2.84)$ \\
\hline Atlantique & $0.69(0.37-1.27)$ & $0.61(0.29-1.29)$ & $0.70(0.28-1.74)$ \\
\hline Borgou & $1.04(0.60-1.80)$ & $0.85(0.44-1.62)$ & $1.15(0.53-2.47)$ \\
\hline Collines & $1.82(0.86-3.86)$ & $0.00(0.00-0.02)^{* * *}$ & $2.48(1.09-5.66)^{* *}$ \\
\hline Couffo & $1.93(0.63-5.88)$ & $0.00(0.00-0.04)^{* * *}$ & $2.99(0.90-9.94)$ \\
\hline Donga & $1.13(0.42-3.08)$ & $0.00(0.00-0.05)^{* * *}$ & $1.42(0.49-4.06)$ \\
\hline Littoral & $0.89(0.44-1.79)$ & $0.79(0.36-1.69)$ & $0.94(0.34-2.57)$ \\
\hline Mono & $0.83(0.42-1.64)$ & $0.47(0.13-1.67)$ & $1.01(0.45-2.24)$ \\
\hline Oueme & $1.09(0.66-1.80)$ & $0.82(0.42-1.59)$ & $1.25(0.63-2.48)$ \\
\hline Plateau & $1.21(0.53-2.76)$ & $0.55(0.12-2.53)$ & $1.59(0.59-4.24)$ \\
\hline Zou & $1.74(1.09-2.77)^{* *}$ & $1.48(0.78-2.82)$ & $0.09(1.03-3.53)^{* *}$ \\
\hline sexe & 0.78 & & \\
\hline
\end{tabular}




\begin{tabular}{llll}
\hline & Univariate analysis & & Male adolescents \\
\cline { 2 - 4 } & Overall & female adolescents & \\
\hline Male & 1 & & 0.84 \\
Female & $1.03(0.79-1.35)$ & 0.58 & 1 \\
Fight & 0.71 & 1 & $1.04(0.69-1.57)$ \\
0 time & 1 & $0.38(0.70-1.83)$ & 0.67 \\
1 or more times & $1.06(0.76-1.48)$ & 1 & 1 \\
Illicite Drug Comsuption & 0.81 & $0.41(0.05-3.11)$ & $1,16(0.57-2.35)$ \\
No & 1 & 0.35 & 0.4 \\
Yes & $1.08(0.55-2.13)$ & 1 & 1 \\
Regular Practice of Physical Activity & 0.25 & $0.83(0.56-1.22)$ & $0.85(0.59-1.23)$ \\
$<3$ days & 1 & 0.61 & 0.83 \\
$3-7$ days & $0.84(0.64-1.12)$ & 1 & 1 \\
Parental supervision & 0.67 & $1.10(0.74-1.63)$ & $1.04(0.70-1.54)$ \\
Never. rarely or sometime & 1 & 0.35 & 0.4 \\
Most of the time or always & $1.06(0.79-1.42)$ & 1 & $0.84(0.57-1.24)$ \\
Parental support & 0.26 & $0.81(0.53-1.24)$ & 0.26 \\
Never, rarely or sometime & & 0.53 & 1 \\
Most of the time or always & $0.84(0.62-1.13)$ & 1 & $0.80(0.55-1.17)$ \\
Relationship with parents/guardians & 0.21 & $0.87(0.58-1.32)$ & \\
Yes & 1 & $0.82(0.61-1.11)$ & \\
No & &
\end{tabular}

Table 3. Continue.

\begin{tabular}{|c|c|c|c|}
\hline & \multicolumn{3}{|l|}{ Multivariate analysis } \\
\hline & Overall & Female adolescents & Male adolescents \\
\hline \multicolumn{4}{|l|}{ Age } \\
\hline$<15$ ans & 1 & 1 & 1 \\
\hline$\geq 15$ ans & $1.66(0.96-2.87)$ & $1.64(0.82-3.25)$ & $1.71(0.76-3.86)$ \\
\hline \multicolumn{4}{|l|}{ Beingbullied } \\
\hline 0 day & 1 & & \\
\hline $1-30$ days & $1.53(1.08-2.17)^{* *}$ & $2.40(1.47-3.93)^{* * *}$ & $1.35(0.86-2.12)$ \\
\hline \multicolumn{4}{|l|}{ Friendship } \\
\hline 0 friend & 1 & 1 & \\
\hline 1 or more & $0.58(0.36-0.94)^{* *}$ & $0.56(0.31-0.99)^{* *}$ & $0.61(0.31-1.19)$ \\
\hline \multicolumn{4}{|l|}{ Class attended } \\
\hline $1^{\text {st }}$ & 1 & 1 & 1 \\
\hline $2^{\text {nd }}$ & $2.08(0.56-7.70)$ & $1.10(0.46-2.61)$ & $3.37(0.52-21.84)$ \\
\hline $3^{\text {rd }}$ & $2.27(0.68-7.66)$ & $1.28(0.57-3.38)$ & $3.44(0.62-18.89)$ \\
\hline $4^{\text {th }}$ & $3.87(1.11-13.55)^{* *}$ & $1.95(0.90-5.48) * *$ & $6.63(1.14-38.57)^{* *}$ \\
\hline $5^{\text {th }}$ & $3.07(0.86-11.07)$ & $0.72(0.26-2.67)$ & $6.05(1.00-36.34)^{* *}$ \\
\hline $6^{\text {th }}$ & $2.19(0.51-9.70)$ & $0.90(0.27-3.91)$ & $3.98(0.48-32.46)$ \\
\hline $7^{\text {th }}$ & $4.71(1.32-16.76)^{* * *}$ & $1.73(0.66-4.53)^{* *}$ & $8.72(1.52-50.08)^{* *}$ \\
\hline \multicolumn{4}{|l|}{ Type of school } \\
\hline \multicolumn{4}{|l|}{ Public } \\
\hline Private & $1.05(0.58-1.88)$ & & $1.33(0.53-3.33)$ \\
\hline \multicolumn{4}{|l|}{ Area of residence } \\
\hline Rural & 1 & 1 & 1 \\
\hline Urban & $0.77(0.53-1.10)$ & $0.53(0.31-0.90)^{* *}$ & $0.82(0.51-1.25)$ \\
\hline \multicolumn{4}{|c|}{ Person with whom the teenager most lived } \\
\hline Father and mother & 1 & & 1 \\
\hline Father & $1.28(0.74-2.22)$ & & $1.40(070-2.77)$ \\
\hline Mother & $1.15(0.76-1.76)$ & & $1.45(0.85-2.47)$ \\
\hline kinships & $1.32(0.65-2.68)$ & & $1.35(0.56-3.22)$ \\
\hline Boarding school & $2.34(1.26-4.35) * *$ & & $2.78(1.40-5.54)^{* *}$ \\
\hline Friends & $0.62(0.21-1.81)$ & & $0.48(0.10-2.21)$ \\
\hline \multicolumn{4}{|l|}{ Alcohol comsuption } \\
\hline 0 day & 1 & 1 & \\
\hline 1 or more days & & $1.14(0.72-1.81)$ & \\
\hline Tobacco comsuption & & & 1 \\
\hline No & 1 & & \\
\hline Yes & $1.57(0.85-2.91)$ & & \\
\hline \multicolumn{4}{|l|}{ Department } \\
\hline Alibori & 1 & 1 & 1 \\
\hline Atacora & 1 & 1 & 1 \\
\hline Atlantique & $0.59(0.25-1.33)$ & $0.82(0.27-2.47)$ & $0.54(0.17-1.67)$ \\
\hline Borgou & $0.95(0.47-1.91)$ & $1.40(0.51-3.81)$ & $0.83(0.32-2.10)$ \\
\hline
\end{tabular}




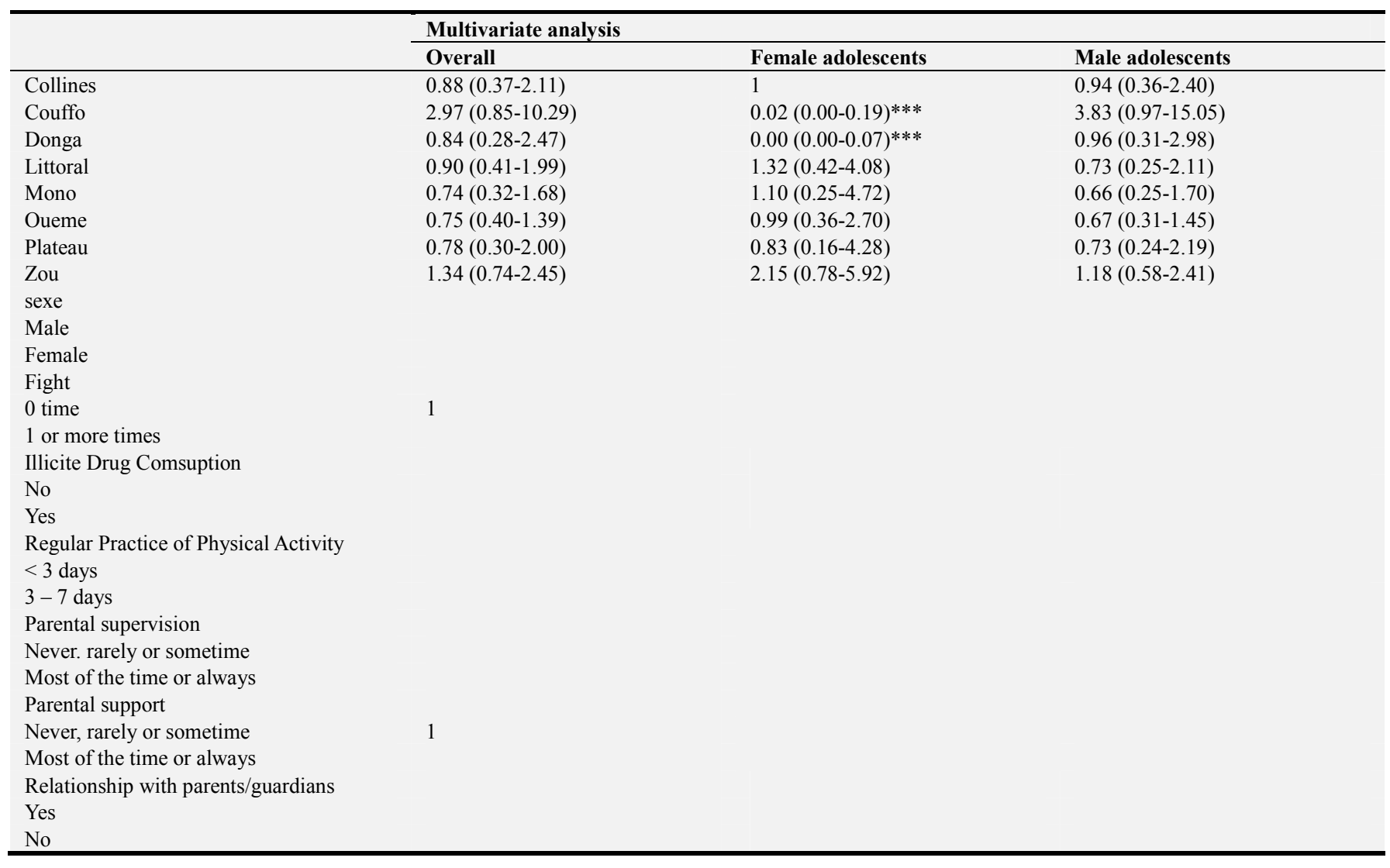

$*=\mathrm{p}<0.05 * *=\mathrm{p}<0.01 * * *=\mathrm{p}<0,001$

\section{Discussion}

To our knowledge, this is the first nationwide study conducted in Benin to examine the prevalence and potential factors associated with psychosocial disorders among adolescents in school.

\subsection{Prevalence}

In our study, the prevalence of psychosocial distress was $10.8 \%$. Some authors have found psychosocial distress prevalences of the same order of magnitude as ours. For example, Zarabi \& al in Gilan in 2009 reported the same prevalence of $10.5 \%$ [12].

Other authors, on the other hand, found higher prevalences than ours in school adolescents. These are Pengpid \& al in Thailand in 2013 [9], Siziya \& al in Zambia in 2015 [10] and Qorbani \& al in Iran in 2014 [13] who reported prevalences of $16.9 \%, 33.5 \%, 15.7 \%$ and $67.7 \%$ respectively. The difference between the Iranian study and ours is that, in the former, seven questions were used to define the psychosocial distress among schooled adolescents assessing: uselessness, anger, anxiety, insomnia, confusion and sadness problems. Respondents who had answered " Yes " to at least four of the seven elements were considered to be psychosocially distressed. However, the definition we used in the present study for psychosocial distress is the one used in Zambia and Thailand's studies [10, 11].

In Quebec in 2001, Breton \& al [14] reported prevalence of psychosocial distress between $18 \%$ and $24 \%$ among adolescents aged 12 to 17 and $29 \%$ among those aged 15 to 19 .

Some authors like Tasmin \& al in 1999 (in children) and Sharifi et al in 2001 in Iran found lower prevalences than ours, $2.1 \%$ and $1.9 \%$ respectively $[15,16]$.

\subsection{Ssociated Factors}

In our study, the prevalence of psychosocial distress was higher among girls under $15(7.9 \%)$ than in boys of the same age range $(4.8 \%)$. This difference related to the age could be explained by the timing of puberty, happening earlier in girls and thought to be associated with an increase in mental health problems [17]. Since girls mature faster than boys in early adolescence, they will be more likely to be psychologically distressed than boys of the same age group.

This same observation was made by Breton \& al in 1999 [14] who reported that the increased incidence of psychological distress among girls is an internationally recognized phenomenon. An epidemiological study conducted in North Carolina among 1420 children and young adolescents indicated a prevalence higher than ours of $17.1 \%$ for girls and $13.0 \%$ for boys. Gasquet \& al in 1994 and Seiffe-Krenke \& al in 2002 found an increase in depression during puberty among girls and a relative stability among boys $[18,19]$. Breton \& al in 1999, as well as Galambos \& al in 2003, estimated that anxiety and depression problems would generally remain stable in adolescence, for boys as much as for girls $[14,20]$. 
Leadbeater \& al found that this phenomenon could be explained by the greater emphasis girls place on interpersonal relationships in comparison to boys [21]. Like Siziya \& al [10], we did not find an association between gender and psychological distress in adolescents. This can be explained by the differences in the stress management strategies boys and girls use, as boys tend to be more detached from their emotions and difficult situations and to be more emotionally inhibited than girls [22].

The difference in prevalence between these studies and ours could be explained by the extent of the study site as well as the targets. Indeed, most of these studies were of regional level or partially covered the adolescence age range while ours was nationwide and covered the whole age range of adolescence. In addition, the difference could also be explained by the sampling techniques and data collection methods used.

The factors associated with psychosocial distress were bullying, friendships, class attended, place of residence, the fact that the teenager had lived in a boarding school.

Adolescents who had been bullied were more likely to develop psychosocial distress than those who had not. This association was also found by Karaman \& al in Turkey in 2006 [22].

Having close friends was a protective factor against psychosocial distress. This result was confirmed by Siziya \& al [10]; close friends should always be there to share their concerns and this would avoid distress. For girls, our study showed the extent of psychosocial distress in rural areas; this can be explained by the social pressures still current in rural areas where girls are often minimized or forced to be quickly socially productive either by getting married or having income-generating activities. Compared to adolescent boys who lived with their parents or guardians, those living in boarding school were more likely to suffer from psychological distress. Separation from parents or guardians would result in emotional deprivation and loss of identification marks in adolescents. Parents are no longer present to listen to the confidences of their children and give them the attention they need. Facing the difficulties of the boarding school, the adolescent will have to wait for days or even months to share his concerns with his parents. This awaiting generates frustration and stress. Picard \& al, however did not report an association between the psychocosial distress and the boarding school [23].

A significant association was also found between psychosocial distress and the class attended by the adolescent. Those who are in examination classes were more likely to suffer from psychological distress and among them boys were more at risk. This association with the classes attended was confirmed by the study of Ge \& al but in the latter, girls were more at risk [24].

Our study did not find an association between age, parental relationship and psychosocial distress. Other authors such as Qorbani \& al. and Siziya \& al. found that adolescents of 15 years and older were at higher risk [10, 13]. Younger adolescents may still be considered as children, so that the community may not be as strict towards them as it is with older adolescents who are considered as adults. For example, older adolescents may have difficulties coping with stimuli, such as cultural values changes and societal roles. Also, older teens may also experience issues related to disappointment, sexual, physical or emotional abuses. Interventions to control distress should however target younger age groups before they are psychosocially distressed. Concerning the parental support, Helsen \& al found that a high parental had a stronger protective influence on the emotional problems of girls than those of boys [25].

\section{Limitations}

For logistical reasons, the study could not be extended to university; this could have led to a selection bias, since adolescents are also at university. However, we are certain that most of teenagers are in secondary schools and very few in universities, which could minimize this selection bias.

The self-administration of the questionnaire might have induced some information biases due to interviewees' negligence or mis - understanding of certain questions. However, we addressed this situation by explaining each question to teens before questionnaire filling.

Also, we may have a desirability bias because some teenagers may not have expressed their feelings or on the contrary amplify what is perceived as acceptable.

The main limitation of this study is its cross-sectional design, which does not make it possible to determine a causal link between the presumed factors of exposure and psychosocial distress. The strength of the present study lies in the large size of the sample, the random sampling mode, which ensures the representativeness of the adolescents attending school.

\section{Conclusion}

Psychosocial distress among adolescents in Benin is a real public health concern. According to the results of this study, the main risk factors were bullying, not having close friends, being in an examination class or final year, residing in rural area and being in a boarding school. The existence of friendly networks of families and close friends is very important. Adolescence is a particularly rich period of life for the building of an individual, but is also a period of great fragility, full of paradox. In case of psychic suffering of adolescents, all parents are likely to encounter difficulties. It appears urgent to put health education back in the families, thereby reducing the risk of psychosocial distress. Knowing the risk factors for psychosocial distress in adolescents will allow a better prevention and promote the mental health of these young adults.

\section{Some Specific Recommendations}

The Ministry of Health needs to increase adolescents parents and communities awareness related to psychosocial 
distress risk factors in adolescents.

Key messages towards parents may include the following:

Avoiding adolescent bullying

Being more attentive to the teenager and avoiding minimizing teens' difficulties because what may seem like a minor problem for an adult can be a major one for an adolescent with possible suicide attempt.

Helping the adolescent increase his self-esteem by encouraging his progress, skills and autonomy and showing him the importance of having positive ideas.

Allowing and even encouraging adolescents in developing their social support, in talking to a resource person about their problems or concerns whether they are a parent, guardian, teacher or close friend.

Learning to know their children for early detection of any unusual behavior from the latter which can be indicative of a dysfunction.

Similarly, the Ministry of Secondary Education, with the support of the Ministry of Health, should sensitize teaching staff to early detection of psychosocial distress in students, particularly those in boarding schools or attending examination classes.

\section{Conflict of Interest}

The authors declare that they have no competing interests.

\section{References}

[1] Bista B, Thapa P, Sapkota D, et al. Psychosocial Problems among Adolescent Students: An Exploratory Study in the Central Region of Nepal. Front Public Health 2016; 4: 7.

[2] Ahmad A, Khalique N, Khan Z, et al. Prevalence of psychosocial problems among school going male adolescents. Indian J Community Med 2007; 32: 219.

[3] Brundtland (Gro Harlem). WHO/The world health report 2001 - Mental Health: New Understanding, New Hope. Genève: World Health Organisation.

[4] Jellinek MS, Murphy JM, Little M, et al. Use of the Pediatric Symptom Checklist to screen for psychosocial problems in pediatric primary care: a national feasibility study. Arch Pediatr Adolesc Med 1999; 153: 254-260.

[5] Polaha J, Dalton WT, Allen S. The Prevalence of Emotional and Behavior Problems in Pediatric Primary Care Serving Rural Children. J Pediatr Psychol 2011; 36: 652-660.

[6] Wittchen HU, Nelson CB, Lachner G. Prevalence of mental disorders and psychosocial impairments in adolescents and young adults. Psychol Med 1998; 28: 109-126.

[7] Keyes CLM. Promoting and protecting mental health as flourishing: a complementary strategy for improving national mental health. Am Psychol 2007; 62: 95-108.

[8] Background-CDC Global School-based Student Health Survey https://www.cdc.gov/gshs/background/index.htm.
[9] Pengpid S, Peltzer K. Bullying and Its Associated Factors among School-Aged Adolescents in Thailand. The Scientific World Journal, 2013, 2013: 1-6.

[10] Siziya S, Mazaba ML. Prevalence and Correlates for Psychosocial Distress Among In-School Adolescents in Zambia. Front Public Health 2015; 3: 7.

[11] Bennett S, Woods T, Liyanage WM, et al. A simplified general method for cluster-sample surveys of health in developing countries. World Health Stat Q Rapp Trimest Stat Sanit Mond 1991; 44: 98-106.

[12] Zarrabi H, Najafi K, Kafi M, et al. Substance Use among Students of Guilan University of Medical Sciences in Iran in 2005-2006. Acta Med Iran 2009; 473-478.

[13] Qorbani M, Kelishadi R, Taheri E, et al. Association between psychosocial distress with cardio metabolic risk factors and liver enzymes in a nationally-representative sample of Iranian children and adolescents: the CASPIAN-III study. $J$ Diabetes Metab Disord 2014; 13: 44.

[14] Breton JJ, Légaré G, Laverdure J, et al. Santé mentale. In: Institut de la Statistique Québec. Québec, 1999, pp. 443-448.

[15] Tasmin F, Psych MRC, Goodman R, et al. The British Child and Adolescent Mental Health Survey 1999: The Prevalence of DSM-IV Disorders.

[16] Sharifi M, Ranjbar M, Shamloo F. Mental health in female pre-university students in Qazvin, Iran, 2001. Dissertation for General Practitioner Degree, Tehran University of Medical Sciences.

[17] Costello EJ, Mustillo S, Erkanli A, et al. Prevalence and development of psychiatric disorders in childhood and adolescence. Arch Gen Psychiatry 2003; 60: 837-844.

[18] Gasquet I. Approche épidémiologique de l'évolution avec l'âge et le sexe de la dépression infanto-juvénile. Psychiatr L'enfant 1994; 27: 533-566.

[19] Seiffe-Krenke I, Stemmler. Factors contributing to gender differences in depressive symptoms: A test of three developmental models. $J$ Youth Adolesc 2002; 31: 405-417.

[20] Galambos NL, Barker ET, Almeida DM. Parents do matter: trajectories of change in externalizing and internalizing problems in early adolescence. Child Dev 2003; 74: 578-594.

[21] Leadbeater BJ, Blatt SJ, Quinlan DM. Gender-Linked Vulnerabilities to Depressive Symptoms, Stress, and Problem Behaviors in Adolescents. J Res Adolesc 1995; 5: 1-29.

[22] Karaman Y, Cinkir S. Bullying among Turkish high school students. Child Abuse Negl 2006; 30: 193-204.

[23] Picard L, Claes M, Melançon C, et al. Qualité des liens affectifs parentaux perçus et détresse psychologique à l'adolescence. Enfance 2007; Vol. 59: 371-392.

[24] Ge X, Conger RD, Elder Jr. GH. Pubertal transition, stressful life events, and the emergence of gender differences in adolescent depressive symptoms. Dev Psychol 2001; 37: 404417.

[25] Helsen M, Vollebergh W, Meeus W. Social support from parents and friends and emotional problems in adolescence. $J$ Youth Adolesc 2000; 319-335. 